\title{
Optimal Dosage and Administration Practices for Vitamin K Antagonist Reversal With 4-Factor Prothrombin Complex Concentrate
}

Clinical and Applied

Thrombosis/Hemostasis

Volume 26: I-I3

(C) The Author(s) 2020

Article reuse guidelines:

sagepub.com/journals-permissions DOI: 10.1 I $77 / 1076029620947474$ journals.sagepub.com/home/cat

@SAGE

\author{
Denise H. Rhoney, PharmD, FCCP, FCCM, FNCS' ${ }^{\circ}$, \\ Katleen W. Chester, PharmD, BCCCP, BCGP ${ }^{2}$, and Damon A. Darsey, MD ${ }^{3}$
}

\begin{abstract}
Expert consensus and international guidelines recommend urgent co-administration of vitamin $\mathrm{K}$ and 4-factor prothrombin complex concentrates (4F-PCCs) to rapidly reverse VKA-related bleeding. This narrative review examined real-world evidence and strategies to optimize international normalized ratio (INR) reversal, hemostasis, and outcomes in patients receiving 4F-PCC in this setting. Key determinants for success include the appropriate use of alternative dosing and administration strategies, such as fixed dosing and increased infusion speed, adherence to institutional guidelines, and removing significant institutional barriers to reduce time to treatment. In the opinion of authors, minimizing the time to treatment with 4F-PCCs is of paramount importance when treating patients with VKA-related bleeding. Practices that safely and feasibly shorten the time to administration should be included in guidelines for institutions responsible for anticoagulant care, and adhered to in centers that perform invasive procedures on patients receiving VKA therapy. Further studies are required to optimize use of 4F-PCC, particularly in relation to the ideal dosing strategy and the role of INR.
\end{abstract}

\section{Keywords}

hemorrhage, warfarin, anticoagulants, prothrombin complex concentrates

\section{Introduction}

Vitamin K antagonists (VKAs), such as warfarin, are prescribed for prevention and treatment of thromboembolic events (TEEs) in many clinical situations; however, VKA treatment is associated with increased bleeding risk. ${ }^{1}$ The goal of urgent reversal of VKA-related bleeding is to rapidly increase the concentration of vitamin $\mathrm{K}$-dependent clotting factors (VKDFs) to reverse international normalized ratio (INR). ${ }^{2}$ This may be achieved by co-administration of vitamin $\mathrm{K}$ with freshfrozen plasma (FFP) or prothrombin complex concentrates (PCCs) (plasma-derived purified products containing coagulation factors [Factors II, VII, IX, and X], plus anticoagulant proteins $\mathrm{C}$ and $\mathrm{S}$, and antithrombin, depending on the product). Clinical differentiators of 4-factor PCCs (4F-PCCs) versus FFP include faster infusion time, less potential for fluid overload, shorter time to administration (no requirement for thawing/ blood-type matching), ${ }^{3}$ and reduced risks of pathogen transmission and allergic reactions. ${ }^{2,4,5}$ In addition, FPP is associated with transfusion-related acute lung injury (TRALI). ${ }^{1}$ TRALI is triggered by the presence of anti-leukocyte antibodies and so is unlikely to occur with 4F-PCCs, as antibodies are removed during the manufacturing process. ${ }^{6,7}$ Current European and US guidelines recommend 4F-PCCs over FFP for rapid reversal of VKA-related bleeding, ${ }^{5,8-13}$ and advocate that centers responsible for anticoagulant care and performing invasive procedures on VKA-treated patients maintain inventory of a licensed 4F-PCC. ${ }^{5}$ Kcentra $^{\circledR}$ (CSL Behring GmbH, Marburg, Germany) is currently the only 4F-PCC approved in the US for urgent VKA reversal in patients with major bleeding or who require urgent surgical/invasive procedures. ${ }^{14}$

\footnotetext{
' UNC Eshelman School of Pharmacy, University of North Carolina, Chapel Hill, NC, USA

${ }^{2}$ Grady Health System, Atlanta, GA, USA

${ }^{3}$ University of Mississippi, School of Medicine, Jackson, MS, USA
}

\section{Corresponding Author:}

Denise H. Rhoney, UNC Eshelman School of Pharmacy, University of North Carolina, Chapel Hill, NC, USA.

Email: drhoney@unc.edu 
Table I. Dosing of Prothrombin Complex Concentrate $\left(\text { Kcentra }^{\circledR}\right)^{14}$.

\begin{tabular}{lccc}
\hline Baseline INR & $\begin{array}{c}\text { IU of coagulation } \\
\text { factor IX (per kg } \\
\text { body weight) }\end{array}$ & $\begin{array}{c}\text { Approximate } \\
\text { dose (mL per kg } \\
\text { body weight) }\end{array}$ & $\begin{array}{c}\text { Maximum } \\
\text { recommended } \\
\text { dose (IU) }\end{array}$ \\
\hline 2 to $<4$ & 25 & 1 & 2500 \\
4 to $\leq 6$ & 35 & 1.4 & 3500 \\
$>6$ & 50 & 2 & 5000 \\
\hline
\end{tabular}

INR, international normalized ratio; IU, international unit.

Although the efficacy and safety of 4F-PCCs are well established for VKA reversal, ${ }^{15-19}$ there is lack of consensus regarding optimal dosing strategies. ${ }^{20}$ On-label dosing of Kcentra is a variable-dose regimen based on bodyweight and pre-treatment INR. It is indicated only for baseline INR $>2$ (Table 1), with pharmacokinetic analysis showing that a $50 \mathrm{IU} / \mathrm{kg}$ dose rapidly increases VKDF concentrations from baseline levels. ${ }^{14} \mathrm{~A}$ drawback of variable dosing is potential administration delays while awaiting pre-treatment INR and bodyweight values. ${ }^{21}$ Indeed, complete reversal of major bleeding should be achieved within $6-8$ hours. ${ }^{5}$ Thus, there is a need to investigate alternative dosing strategies for emergency situations. Several studies have investigated fixed dosing ${ }^{22}$ and an American College of Cardiology (ACC) Expert Consensus paper supported use of 2 fixed 4F-PCC doses (1000 IU for reversal of major bleeding; $1500 \mathrm{IU}$ for intracranial hemorrhage [ICH]), in addition to supporting standard-of-care variable dosing. ${ }^{12}$ However, there are concerns that these protocols may lead to under-dosing, which could result in suboptimal responses.

The methods of assessing the effectiveness and clinical utility of a dosing regimen can vary. Assessment of clinical hemostasis attainment and clinical outcomes is most commonly employed, although coagulation testing (particularly the proportion of patients achieving a target INR in a given time) may also be used, together with occurrence of adverse events (thromboembolic or otherwise) and cost.

This narrative review examined real-world strategies to optimize INR reversal, hemostasis, and outcomes in patients receiving 4F-PCC for reversal of VKA-related bleeding, focusing on alternative dosing strategies, infusion speed and overcoming institutional barriers to effective, timely reversal of VKA-related bleeding in clinical practice.

\section{Methods}

This publication is not intended as a systematic review. A PubMed search was conducted to identify publications on PCC administration practices and reversal of VKA-related bleeding, published between January 1, 2013 and December 31, 2019. Search terms are provided in Table 2. The results were filtered for English language papers, and screened for relevance to the administration of 4F-PCC in emergency VKA reversal settings (excluding those that use only on-label variable dosing strategies, where dosing strategy is not specified, or involve direct
Table 2. Search Parameters for Literature Review.

\begin{tabular}{l}
\hline Search parameters \\
\hline Date - Publication: 20I3/0I/0I and 20I9/I2/3I \\
Kcentra (OR Beriplex OR Confidex) AND administration; Kcentra \\
(OR Beriplex OR Confidex) AND dose; prothrombin complex \\
concentrate*AND dosing; prothrombin complex concentrate*AND \\
dose; prothrombin complex concentrate* AND administration; \\
prothrombin complex concentrate* AND rural OR remote \\
\hline
\end{tabular}

oral anticoagulation therapy rather than VKAs). Preclinical studies and reviews were excluded. Abstracts from congresses meeting relevance criteria were also included. In total, 54 relevant publications were included.

\section{Results}

\section{Alternative Dosing Strategies}

Studies providing information on all alternative dosing strategies (including fixed dosing, use of low variable doses $[\leq 25$ $\mathrm{IU} / \mathrm{kg}$ ], repeat dosing and non-INR-based approaches) are described in Table 3.

Fixed dosing. Although the approved on-label dosing of 4FPCCs is a variable regimen dependent on baseline INR and bodyweight, a number of studies have investigated fixed doses for urgent reversal of VKA-related bleeding. The minimum effective and maximum tolerable doses have also been considered. A retrospective study of 103 patients requiring anticoagulation reversal (VKA-related bleeding or prior to an invasive procedure) assessed efficacy of fixed-dosing of 1000 IU 4FPCC by clinical hemostasis, as well as INR reversal. Excellent clinical response (control of bleeding without requiring additional measures) was seen in $84 \%$ of patients. The authors concluded that 1000 IU fixed-dosing appeared effective and well tolerated. ${ }^{25}$ Results from another retrospective review of 39 patients ${ }^{23}$ concluded that a single, fixed dose of 1500 IU 4FPCC effectively reduced median INR in $92 \%$ of patients with VKA-related bleeding, with no associated TEEs within 7 days. A single, fixed dose of 1500 IU 4F-PCC was also shown to reduce median INR in $100 \%$ of patients with VKA-related bleeding or undergoing urgent surgery $(\mathrm{n}=37)$ in another retrospective chart review. ${ }^{24}$ In addition, there were no early or late thrombotic complications, or immediate redosing requirements, though 1 patient required a repeat 4F-PCC dose 2 days after the initial dose. ${ }^{24}$ While the latter 2 studies support fixed dosing, neither included endpoints for clinical outcomes (e.g. bleeding cessation).

A recent study evaluated the frequency with which an initial fixed 4F-PCC dose of $1500 \mathrm{IU}$ was insufficient to achieve an indication-specific INR ( $\leq 1.4$ for central nervous system [CNS] bleeds and $<2$ for all other indications) in patients requiring warfarin reversal. ${ }^{26}$ Overall, only $11 / 63$ patients (17\%; including 8 patients with CNS bleeds) were eligible to receive a supplemental dose based on failure to achieve their 


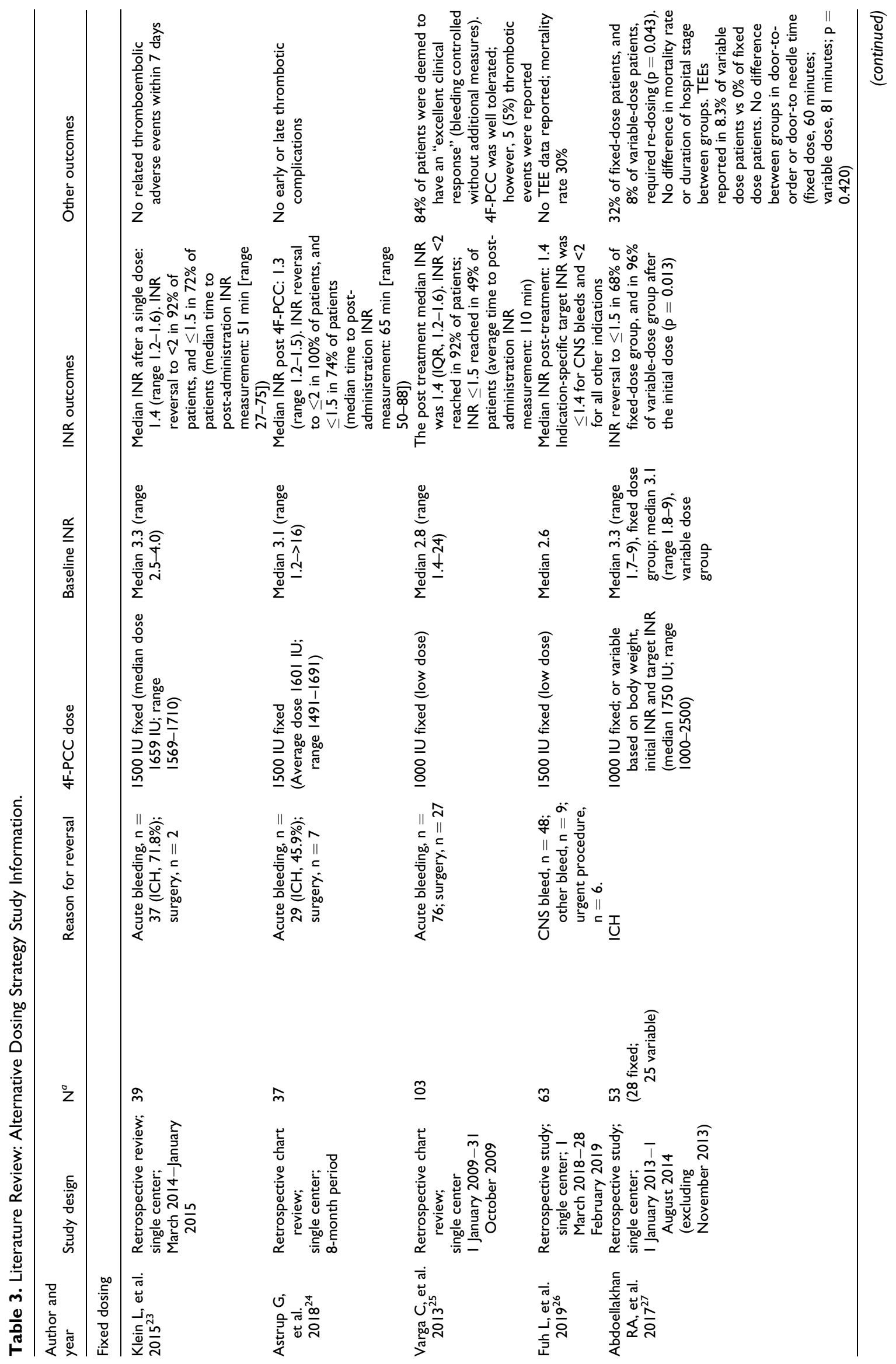




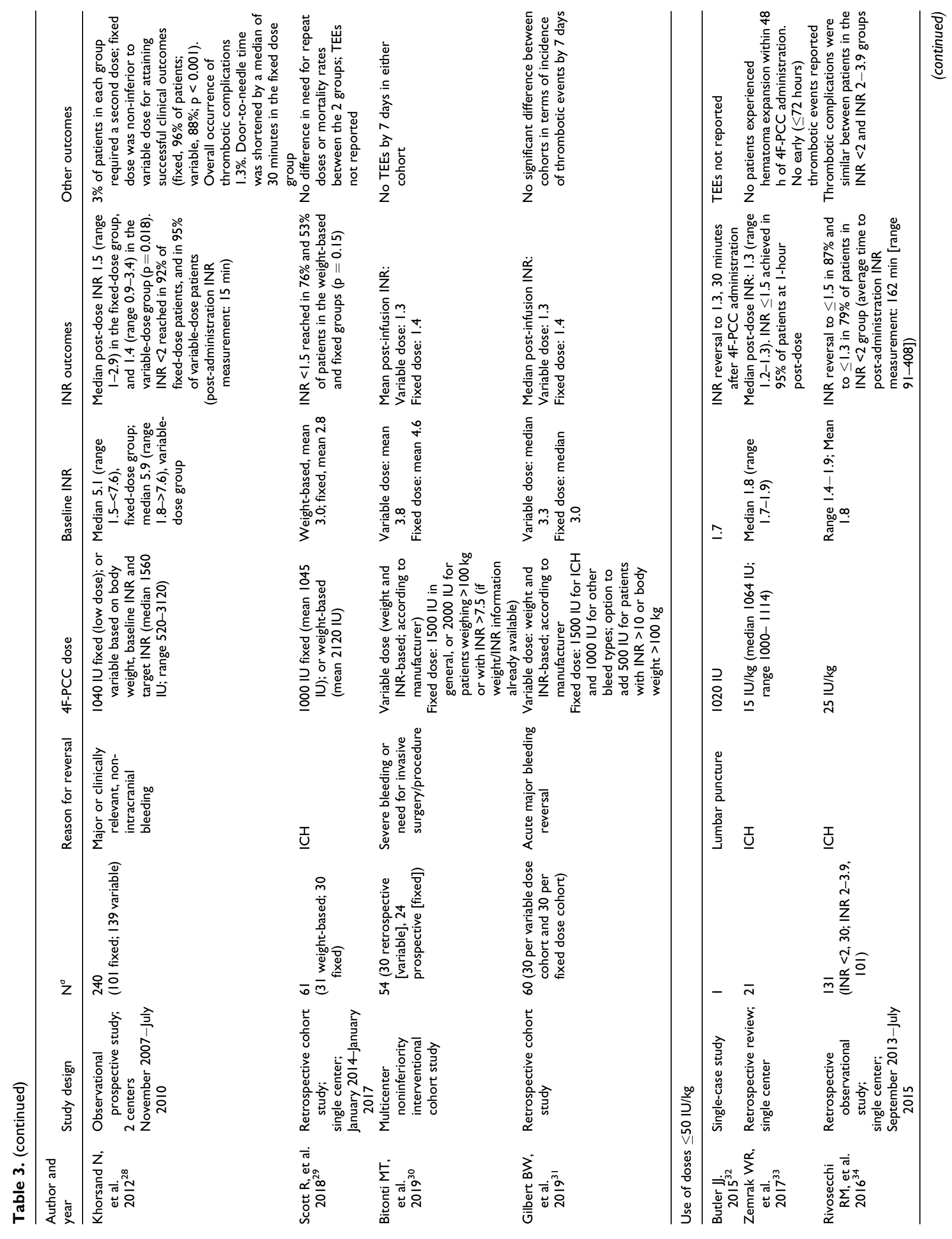




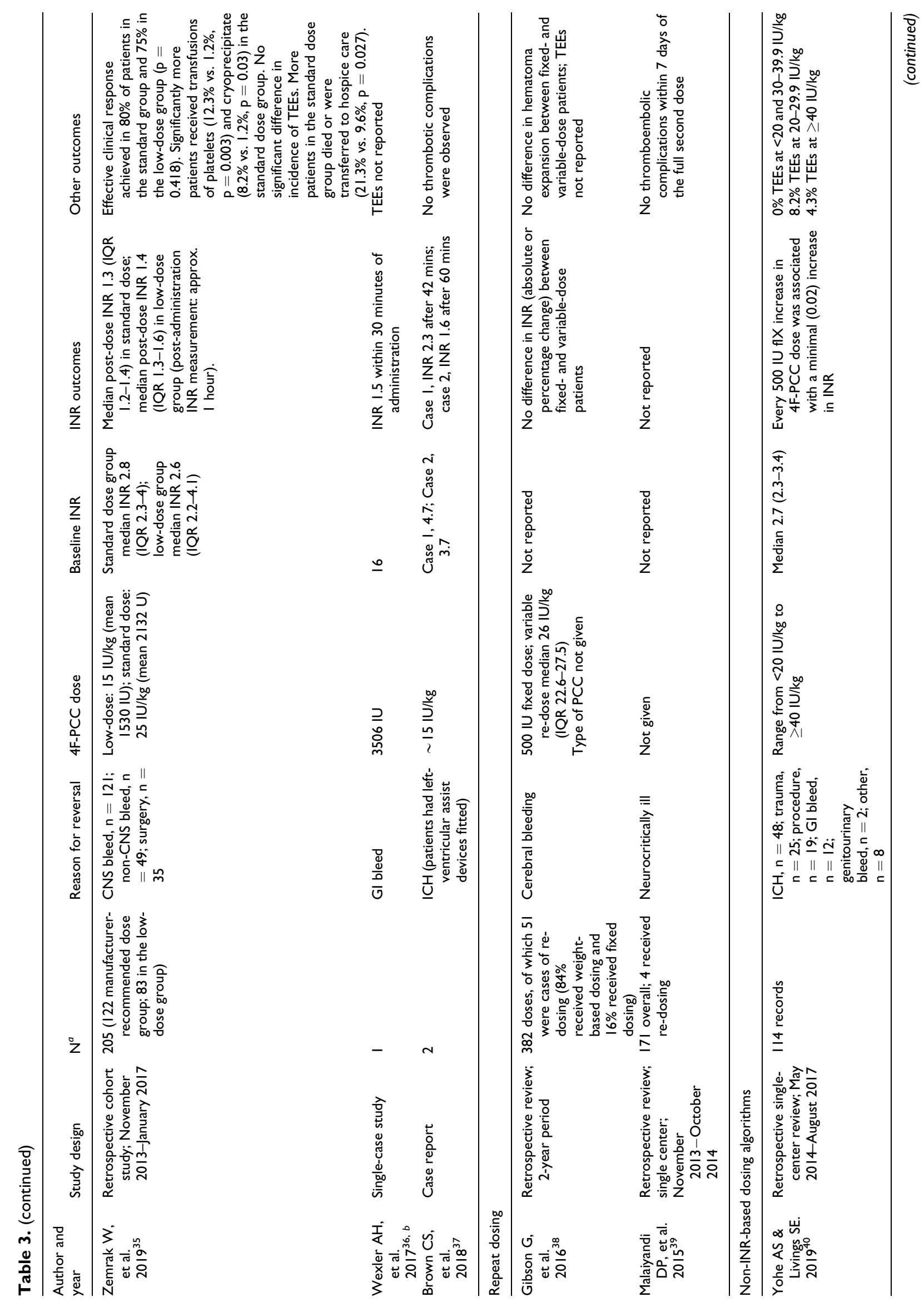




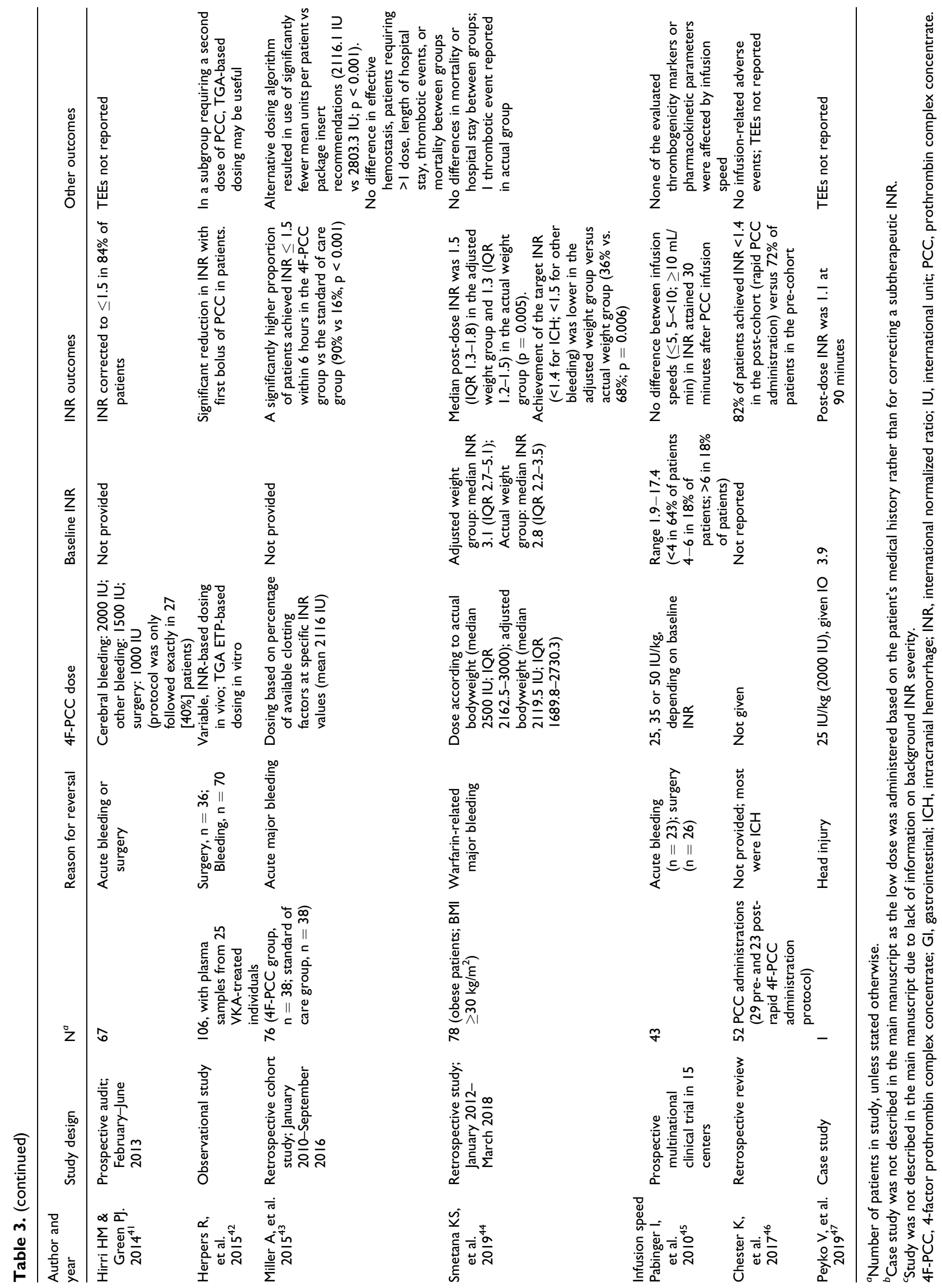


INR goal (i.e. 4F-PCC 1500 IU was inadequate). However, the supplemental dose was actually administered in only $2 / 11$ patients, calculated as the remainder of the standard weightand INR-based dosing (both of these patients had an initial INR $>3.5$ ). The authors concluded that a low fixed dose of $1500 \mathrm{IU}$ 4F-PCC was sufficient for achievement of INR goals in the majority of patients. ${ }^{26}$

Additionally, studies have been conducted to compare the use of fixed and variable 4F-PCC dosing. Abdoellakhan et al. conducted a retrospective study comparing 28 patients with VKA-related ICH receiving a fixed dose of 1000 IU 4F-PCC with 25 patients receiving the on-label variable dose based on body weight, initial INR and target INR. ${ }^{27}$ In the fixed-dose group, median INR decreased in $68 \%$ of patients, versus $96 \%$ of patients in the variable-dose group after the initial dose $(\mathrm{p}=$ 0.013). There was no significant difference in median "door-toneedle" administration interval between dosing groups. It was concluded that $1000 \mathrm{IU}$ was insufficient for reversal of VKArelated ICH.

An observational prospective study over nearly 3 years in 240 patients requiring $4 \mathrm{~F}-\mathrm{PCC}$ for major non-intracranial bleeding demonstrated INR $<2$ achievement in $92 \%$ of patients receiving fixed-dose 1040 IU 4 F-PCC versus $95 \%$ of patients in the variable-dose group, based on weight and INR. ${ }^{28}$ This study failed to demonstrate non-inferiority by the INR outcome. However, a successful clinical outcome (cessation of visual bleeding, no further decrease in hemoglobin, normalized blood pressure, and no further need for PCC or blood transfusion) was judged by the attending physicians in $96 \%$ and $88 \%$ of the fixed- and variable-dose groups, respectively. The fixeddose strategy was non-inferior to the variable regimen, in terms of clinical outcome. Notably, the administration interval was shortened in the fixed cohort by a median of 30 minutes.

Another retrospective study, which included 61 patients with VKA-related ICH, found no significant difference in INR reduction or INR target achievement between fixed-dose 1000 IU PCC and on-label 4F-PCC dosing based on bodyweight. INR $<1.5$ was achieved in $53 \%(16 / 30)$ who received fixed dosing and $71 \%(22 / 31)$ of patients receiving a weight-based 4F-PCC dose $(\mathrm{p}=0.15) .{ }^{29}$ It should be noted that this study had low patient numbers and was underpowered to show a difference between strategies, and also did not investigate hemostatic efficacy. The authors concluded that an optimal dosing strategy for 4F-PCC may involve accounting for both weight and initial INR.

A recent study compared variable-dose 4F-PCC treatment (based on weight and INR) and fixed-dose 4F-PCC treatment (1500 IU, or $2000 \mathrm{IU}$ in specific populations [i.e. patients weighing $>100 \mathrm{~kg}$ or with a baseline INR $>7.5]$ ) respectively. A total of 30 patients were included in the retrospective cohort (variable dose) and 24 in the prospective cohort (fixed dose). ${ }^{30}$ Overall, achievement rates for post-infusion INR $<2$ (primary endpoint) were similar for both cohorts $(96.7 \%$ for variable dose vs $95.8 \%$ for fixed dose; $p=0.0035$ for non-inferiority). A greater proportion of patients in the variable dose cohort achieved a post-infusion INR $<1.5$ compared with the fixed- dose cohort ( $90 \%$ vs $75 \%$; $p>0.45$ for non-inferiority). This observation may be linked to the lower mean baseline INR observed in the variable-dose cohort compared with the fixed-dose cohort (3.8 vs 4.6). ${ }^{30}$ Another recent study compared on-label variable-dose 4F-PCC and low fixed-dose approaches (1500 IU for ICH; $1000 \mathrm{IU}$ for other bleed types) in 60 patients requiring acute warfarin reversal (30 per dosing strategy). Target post-infusion INR in this study was $<1.6$ (primary endpoint). There was no significant difference between treatment groups in number of patients achieiving INR $<1.6$ or $<1.4$ following 4F-PCC infusion. ${ }^{31}$

Use of doses $\leq 50 \mathrm{IU} / \mathrm{kg}$. While results from these studies generally support fixed dosing with $\geq 1000$ IU $4 F-P C C$ if the baseline INR for reversal is $>2$, few data have been published on $4 \mathrm{~F}-\mathrm{PCC}$ for reversal in patients with baseline INRs $<2$; there is currently no indicated dose of Kcentra for this particular population. ${ }^{14} \mathrm{~A}$ case study reported that administration of lower than approved weight-based variable-dose 4F-PCC (10 IU/kg; total, $1020 \mathrm{IU}$ ) reduced INR to $\leq 1.5$ within 30 minutes in a patient requiring VKA reversal for a lumbar puncture (although multiple FFP units were initially used to reverse INR from $>3$ to 1.7$){ }^{32}$ Supporting low variable-dose regimens for patients with low INRs, $15 \mathrm{IU} / \mathrm{kg}$ 4F-PCC given to 21 patients with warfarin-associated ICH and baseline INRs ranging from 1.6 to 1.9 , reversed INR to $\leq 1.5$ in $95 \%$ of patients, with no hematoma expansion within 48 hours of dosing and no TEEs within 72 hours. ${ }^{33}$ In another study in ICH, of 30 patients with low baseline INRs receiving $25 \mathrm{IU} / \mathrm{kg} 4 \mathrm{~F}-\mathrm{PCC}, 87 \%$ achieved INR $\leq 1.5 .^{34}$ Finally, a retrospective observational study in 205 patients with warfarin-related bleeding found fewer patients met the primary outcome of INR reversal plus effective clinical response in the low variable-dose 4F-PCC group (15 IU/kg; $61.4 \%)$ versus the on-label variable-dose 4F-PCC group (25 $\mathrm{IU} / \mathrm{kg} ; 75.4 \%, \mathrm{p}=0.033) .{ }^{35}$ Baseline INR values were 2.6 in the low variable-dose group and 2.8 in the on-label variabledose group. This result was primarily driven by the lower number reaching the target INR; there was no difference in rates of effective clinical response and patients in the low variable-dose group had lower mortality. Overall, these studies suggest that low variable-dose 4F-PCC may suffice for urgent reversal of modest INR elevation and management of bleeding.

Additionally, low variable doses of 4F-PCC may be considered in patients at high risk of thrombosis to minimize the risk of TEEs. For example, a case report of a patient at high risk of TEEs who presented with a life-threatening gastrointestinal bleed found that a $35 \mathrm{IU} / \mathrm{kg}$ dose of 4F-PCC successfully lowered INR from 16 to 1.5 at 30 minutes post administration. ${ }^{36} \mathrm{~A}$ case report of 2 patients with left-ventricular assist devices who developed warfarin-related ICH reported that $\sim 15 \mathrm{IU} / \mathrm{kg} 4 \mathrm{~F}-$ PCC successfully reversed INR with no thrombotic complications. ${ }^{37}$ Furthermore, in the retrospective observational study described above, no significant difference in TEEs were observed between $25 \mathrm{IU} / \mathrm{kg}$ and low variable-dose $4 \mathrm{~F}-\mathrm{PCC}$ $(15 \mathrm{IU} / \mathrm{kg})$ with 72 hours $(4.1 \%$ vs $1.2 \%$; $=0.404) .{ }^{35}$ Overall, 
given the lack of data, further examination of low variable-dose $4 \mathrm{~F}-\mathrm{PCC}$ strategies is warranted.

Repeat dosing. A lack of clinical data on the safety and efficacy of repeat dosing with 4F-PCCs means that labeling advises against re-dosing. ${ }^{14}$ However, an international survey of emergent warfarin reversal strategies that included members of the Biomedical Excellence for Safer Transfusion Collaborative, found that most surveyed clinical sites use post-PCC INR values as guidance for 4F-PCC repeat dosing, indicating the real-world practice of re-dosing. ${ }^{48}$ In the aforementioned Abdoellakhan et al. study comparing 4F-PCC fixed- versus variable-dosing in patients with VKA-related ICH, repeat dosing was needed in $32 \%$ and $8 \%$ of fixed- and variable-dose patients, respectively, to achieve INR $\leq 1.5 .^{27}$ In 51 recorded cases of repeat dosing, no difference was observed in INR (absolute or percentage change) or hematoma expansion in patients with cerebral bleeding receiving variable weight-based PCC redosing (median $26 \mathrm{IU} / \mathrm{kg}$ ), or fixed $500 \mathrm{IU}$ re-dosing (type of PCC not reported). ${ }^{38}$ Furthermore, TEEs, the main risk associated with re-dosing, were not observed in the 7 days following a full second 4F-PCC dose in 4 neurocritically ill patients identified from a retrospective review of 171 patients receiving 4F-PCC. The authors concluded that 4F-PCC re-dosing might be well tolerated in such patients, although larger prospective studies are needed to support these findings. ${ }^{39}$

Non-INR-based dosing algorithms. Pre-treatment INR is widely used to estimate the 4F-PCC dose needed to achieve target INR, either alone or in consideration with bodyweight (Table 1). Apart from fixed dosing, few alternatives to the standard-of-care algorithms for dosing and monitoring anticoagulant therapy have been investigated, despite a 2-year multicenter study in 256 patients concluding that adequate reversal of VKA-related bleeding does not depend on pre-treatment INR. ${ }^{49}$ A retrospective review of 114 medical records for patients receiving variable-dose 4F-PCC (dosed based on bodyweight and baseline INR) for reversal of warfarin is also of relevance. A significant linear relationship was observed between 4F-PCC dose and post-dose INR, but bodyweight and baseline INR were not correlated with post-dose INR. These results support alternative 4F-PCC dosing strategies, independent of bodyweight and baseline INR. ${ }^{40}$

Alternative dosing algorithms have been proposed, including regimens based on bodyweight alone, or the indication for administration. ${ }^{41}$ Indeed, an audit of warfarin reversal assessing a 3-level dosing protocol (2000 IU for CNS bleeds, 1500 IU for other bleeds and 1000 IU prior to invasive procedures) found the INR was reversed to $\leq 1.5$ in $84 \%$ (56/67) of patients, suggesting a simple protocol can be effective. However, adherence to the protocol was poor, as the medical team did not regularly consult it in emergency situations (the protocol was followed exactly in only $40.2 \%$ of patients). ${ }^{41}$

A thrombin generation assay (TGA)-based calculation is an experimental approach to monitoring VKA treatment, bleeding risk, and 4F-PCC reversal of VKA-related bleeding. TGA- based calculations may be superior to INR-based calculations (based on the linear relationship between hemorrhagepredictive TGA parameters and 4F-PCC dose for the whole therapeutic range versus the exponential relationship demonstrated for INR and dose). ${ }^{42}$ TGA-based algorithms cannot, however, replace INR-guided dosing in practice, because calculations based on assays are unsuitable when urgent INR reversal is needed. Although this approach is promising, due to various challenges (including its time-consuming nature and the lack of official standardization or reference values), it requires further study before it can enter routine clinical use. ${ }^{50}$

A hematologist-derived strategy is another algorithm for guiding 4F-PCC dosing, which is based on the percentage of available clotting factors at specific INR values. This strategy was examined in a retrospective cohort study of 76 patients and showed that a higher proportion of patients achieved INR $\leq 1.5$ within 6 hours of infusion versus patients treated with an older standard-of-care protocol including products such as FFP, vitamin $\mathrm{K}$ and 3F-PCC (90\% versus $16 \% ; \mathrm{p}<0.001) .{ }^{43}$ Patients dosed according to this alternative algorithm also required significantly fewer units of $4 \mathrm{~F}-$ PCC compared with on-label variable dosing (2116 IU versus $2803 \mathrm{IU} ; \mathrm{p}<0.001)$. These results suggest this alternative 4FPCC dosing may be effective for VKA reversal; however, there was no difference between groups in hemostasis restoration, redosing, hospital stay duration, or adverse events.

The appropriate dosing strategy for 4F-PCC in obesity has also been questioned. A retrospective study investigated the rate of successful INR reversal in 78 obese patients (BMI $\geq 30 \mathrm{~kg} / \mathrm{m}^{2}$ ) experiencing warfarin-related major bleeding when dosed based on adjusted bodyweight compared with actual bodyweight as a cost-saving initiative. Achievement of the target INR was lower in the adjusted weight group versus actual weight group (36\% vs $68 \%$; $p=0.006$ ), with no differences in mortality or hospital stay, indicating 4F-PCC should be dosed according to the manufacturer's guidelines in obese patients until further data are available. ${ }^{44}$

\section{Increasing Infusion Speed}

Faster infusion speeds are a key advantage of PCCs over FFP for VKA-related bleeding reversal. ${ }^{3}$ The maximum recommended 4F-PCC infusion speed is $8.4 \mathrm{~mL} / \mathrm{min}$ (Kcentra) ${ }^{14}$ this cap reflects concerns that more rapid infusion of coagulation factors could trigger TEEs or infusion-related reactions. However, a clinical trial in 43 patients with acute bleeding, or requiring INR reversal for emergency surgical or invasive diagnostic interventions, showed neither an increase in peak levels of thrombogenicity markers, nor greater cumulative elevation, when a median volume of $90 \mathrm{~mL} 4 \mathrm{~F}-\mathrm{PCC}$ was administered at up to $40 \mathrm{~mL} / \mathrm{min}$, versus as low as $2 \mathrm{~mL} / \mathrm{min} .{ }^{45}$ Patients in this study had diverse INRs, age, indications, and BMI, so were representative of patients undergoing reversal of VKA-related bleeding in clinical practice. Infusion speed was not determined by baseline variables, but determined at the investigator's discretion. Ultimately, increasing infusion speed did not affect the INR achieved 30 minutes postinfusion, and the 
authors concluded that 4F-PCC could be rapidly infused for effective emergency reversal of VKA-related bleeding. ${ }^{45}$ This conclusion is supported by a retrospective study in which rapid intravenous push administration of 4F-PCC (mostly for ICH, maximum infusion speed of approximately $750 \mathrm{IU} / 15 \mathrm{~mL}$ per minute) was well tolerated and effective, although time to administration was longer after implementation of the rapid 4F-PCC administration protocol, possibly due to operational limitations such as additional clarification and approval of orders resulting from the use of 4F-PCC in non-standard locations and off-label indications. ${ }^{46}$

\section{Alternative Administration Routes}

A case study reported successful intraosseous (IO) administration of 4F-PCC when several attempts for IV access failed in a warfarin patient reporting to the emergency department (ED) with a head injury. INR was reduced from 3.9 to 1.1 at 90 mins after 4F-PCC dosing ( $25 \mathrm{IU} / \mathrm{kg})$. These results suggest that IO 4F-PCC may be a viable alternative route in an emergency situation to avoid delay in treatment when IV access has not been established. ${ }^{47}$

\section{Removing Institutional Barriers to Urgent 4F-PCC Administration}

While several guidelines recommend the use of 4F-PCC for the urgent reversal of VKAs, ${ }^{5,8-13}$ significant logistic-, therapy-, or clinician-related barriers and lack of institution-specific treatment protocols represent potential impediments to effective, timely reversal of VKA-related bleeding. ${ }^{51}$ In turn, this impedes the attainment of optimal patient outcomes.

Reducing treatment delays. Early intervention underlies effective VKA reversal; thus, time to $4 \mathrm{~F}-\mathrm{PCC}$ administration, also known as "door-to-needle time", is an important determinant of patient outcomes. The time-critical nature of INR reversal was demonstrated in a retrospective cohort study of 853 patients with VKA-related ICH, of whom 307 experienced hematoma enlargement (median baseline INR 2.80 [IQR 2.30-3.41]) and $546 \mathrm{did}$ not (median baseline INR 2.68 [IQR $2.23-3.38]$ ). Achievement of INR $<1.3$ within 4 hours of admission was associated with lower hematoma enlargement rates than when not achieved (43/217 [20\%] vs 264/636 [42\%]; $\mathrm{p}<0.001) .^{52}$ In addition, a prospective study of 822 patients with VKA-related severe hemorrhage showed that 4F-PCC administration concordant with international guidelines (dose $\geq 20 \mathrm{IU} / \mathrm{kg}$, together with vitamin $\mathrm{K}$ ) within 8 hours of patient admission was associated with a greater proportion of patients achieving post-reversal INR $\leq 1.5$ (mean baseline INR 4.7) than the non-concordant group. ${ }^{53}$ Strategies to reduce the 4F-PCC administration interval include fixed dosing, ${ }^{22}$ and, potentially, increasing 4F-PCC infusion speed. ${ }^{45,46}$ Stocking 4F-PCC in high-use areas, such as EDs, has also been considered, as this could increase access to 4F-PCC and allow pharmacist preparation by the bedside compared with the central pharmacy. However, it remains to be seen whether this approach would be effective in reducing administration interval. ${ }^{54,55}$

Targeting institutional delays is another key way in which door-to-needle time can be reduced. In a retrospective study of patients with ICH undergoing VKA-related bleeding reversal, delays to rapid administration of 4F-PCC included hematology consultation, collection of 4F-PCC from the pharmacy or blood transfusion service, and obtaining patients' INR results. ${ }^{56}$ Potential solutions to these barriers include point-of-care INR testing, ${ }^{48}$ allowing ED doctors to request and prescribe $4 \mathrm{~F}$ PCCs without the hematologist's prior approval, expediting delivery of 4F-PCC from the blood transfusion service or pharmacy, and adopting pharmacy verification and dispensing of 4F-PCCs. ${ }^{3,57-60}$ Indeed, it was shown that a pharmacist-driven protocol (in which the pharmacist calculated the dose, prepared 4F-PCC and delivered it to the bedside) reduced the administration interval to 35 minutes versus 70 minutes for the blood transfusion service-driven protocol. However, the impact of this reduction on, INR correction/ICH progression was not reported. ${ }^{54}$

Similarly, a retrospective cohort study including 252 patients receiving 4F-PCC for life-threatening bleeding or urgent surgery in the ED found the median time to 4F-PCC administration was significantly shorter when a pharmacist was present at the bedside compared with physician-only teams (66.5 vs $206.5 \mathrm{~min} ; \mathrm{p}<0.001)$. Although no difference in hemostasis or mortality was observed, patients with a pharmacist at the bedside had a shorter hospital stay. However, it should be noted that patients on various forms of oral anticoagulation were included in this study. ${ }^{61}$ In support of these findings, another retrospective study of patients with bleeding (the majority of whom received warfarin) found that the time from order to start of administration of PCC (3F- or 4F-PCC) was significantly reduced when an ED pharmacist was involved (median $24 \mathrm{~min}$ [IQR 15-35] vs $42 \mathrm{~min}$ [IQR 32 59]; $\mathrm{p}<0.001) .{ }^{3}$ Furthermore, the implementation of an electronic order set for 4F-PCC for reversing VKA-related ICH demonstrated a significant reduction in the door-to-needle time from 83 to 45 minutes $(\mathrm{p}=0.02)$ and a significant improvement in patients receiving the target dose (pre-order set, $29.4 \%$ vs post-order set, $92.9 \%[\mathrm{p}=0.001])^{62}$

Further consideration could be given to early, pre-hospital, administration of 4F-PCCs. This practice is currently quite rare, but could have a substantial impact. ${ }^{63}$ Pre-hospital administration of 4F-PCCs by a mobile stroke unit, ${ }^{64}$ air ambulance ${ }^{63}$ or emergency retrieval service, ${ }^{65}$ particularly in remote settings where access to definitive care may otherwise be unavailable or limited, ${ }^{66}$ should also reduce door-to-needle time versus waiting until hospital presentation. ${ }^{67}$ Such early intervention has been shown to significantly increase the proportion of patients achieving a target INR of $\leq 1.5$ from 3.7 upon ED arrival (proportion difference $0.8 ; \mathrm{p}<0.0001$ ), and faster overall time to normalization (181 versus 541 minutes with no early intervention; $\mathrm{p}<0.001) .{ }^{63}$ Similarly, a retrospective cohort study comparing patients with warfarin-associated ICH who had transferred from a local community hospital $(\mathrm{N}=177)$ or 
presented directly to the $\mathrm{ED}(\mathrm{N}=26)$, found the time to administration of 4F-PCC was longer in transferred patients (median difference $-176 \mathrm{~min} ; \mathrm{p} \leq 0.001$ ). However, the delay was not associated with worse patient outcomes, with no difference in hematoma expansion, hospital stay, or mortality. ${ }^{68}$

Administration of 4F-PCC may also be delayed as a result of dispensing practices at each institution. ${ }^{69}$ 4F-PCC is supplied in vials (500/1000 IU) and requires reconstitution with sterile water. The required dose can be dispensed as described in each package insert, or it can be administered per vial (the amount rounded to allow for use of an exact number of vials). The former option requires an extra step to check the exact contents of each box, reducing waste but potentially resulting in delays in administration. ${ }^{69}$

Adherence to institutional guidelines. VKA-related bleeding should be managed according to institutional protocols based on international and expert guidelines that consider the urgency of the clinical situation, assessment of coagulation (location, extent, and severity of bleeding), laboratory testing, reversal agent selection, and dosing strategy. ${ }^{5,8-13}$ Despite the lifethreatening consequences of acute bleeding, not all EDs have internal protocols guiding anticoagulant reversal, ${ }^{70}$ potentially resulting in poor attainment of target INRs and hemostasis. Implementation of an institutional protocol involving 4FPCC, accompanied by an education program for the clinical team to align reversal strategies, ${ }^{70}$ has the potential to reduce door-to-needle times. ${ }^{71,72}$ This should translate to improved INR reversal and decreased mortality,${ }^{53}$ as well as potentially reduced use of FFP. ${ }^{73}$

\section{Discussion}

Life-threatening VKA-related bleeding or VKA reversal prior to urgent surgery requires emergency treatment, which can be treated via withholding VKAs, and administering 4F-PCC with vitamin $\mathrm{K}$. Controlled clinical trials of 4F-PCC dosing and administration practices in patients with life-threatening VKA-related bleeding are challenging because of difficulties obtaining patient consent in emergency situations where delays cost lives. A consensus on optimal practices is therefore informed by real-world 4F-PCC use, ranging from expert opinions to anecdotal case studies and retrospective reviews. Indeed, most of the studies identified in our literature search are retrospective and observational in nature. While limited, current literature generally supports fixed dosing, ${ }^{12,22,28,74}$ with rationale centered on the critical time saved by eliminating weight- and INR-based calculations, and the potential costeffectiveness of a fixed-dosing approach. ${ }^{41,74}$ A comprehensive review of fixed dosing studies published from the earliest available date in PubMed through 2018, with doses ranging from 200 to $2000 \mathrm{IU}$, supported a fixed-dose strategy of 1000-1500 IU. However, higher doses may be needed to treat ICH or a higher baseline INR. ${ }^{75}$ Nevertheless, in the absence of an ideal dose suitable for all, variable dosing per label instructions is preferred by many institutions.
An important limitation of many studies to date is the use of INR as a surrogate marker of VKA reversal as a key efficacy endpoint. A direct measure of clinical hemostasis/bleeding cessation may be a more clinically relevant outcome. Furthermore, with inconsistent definitions of successful clinical outcomes and safety endpoints, questions remain regarding the minimum effective and maximum tolerable 4F-PCC doses, which respectively carry risks of under- and over-dosing. The ongoing PROPER3 study, a randomized controlled trial comparing fixed vs variable doses of 4F-PCC using hemostatic efficacy as the primary endpoint and INR reversal as the secondary, will hopefully provide further information on the efficacy and safety of these dosing regimens. ${ }^{76}$

In addition, further studies are needed to adequately assess the importance of pre-treatment INR for determining 4F-PCC dose, complemented by studies into alternative algorithms and means of individualized dosing. Furthermore, outcomes of large safety and efficacy re-dosing studies are needed to validate a repeat-dose approach, given the narrow margin between the therapeutic and adverse effects of 4F-PCCs.

Time to treatment is also a key determinant of successful INR reversal and patient outcomes, and can be expedited by pre-hospital administration by mobile units or emergency services, and improvements in hospital logistics. ${ }^{56,63,64}$ Logistical improvements could include point-of-care INR testing, ${ }^{48}$ clinically appropriate administration of 4F-PCCs by ED doctors or pharmacists, ${ }^{3,57-59,61}$ and adopting strategies to improve 4FPCC access. ${ }^{55}$ Implementation of institutional guidelines endorsing autonomous verification and dispensing of 4FPCCs are associated with better patient outcomes, including INR reversal and hemostasis control, ${ }^{3,57-59}$ as are guidelines providing clear recommendations on 4F-PCC dosing and administration practices, even if guidance contravenes label recommendations. ${ }^{53}$

In the opinion of authors, minimizing the time to treatment with 4F-PCCs is of paramount importance when treating patients with VKA-related bleeding. Practices that safely and feasibly shorten the time to administration should be included in guidelines for institutions responsible for anticoagulant care, and in centers that perform invasive procedures on patients receiving VKA therapy.

In summary, there are various key determinants contributing to successful INR reversal, hemostasis, and outcomes in patients receiving 4F-PCC to rapidly reverse VKA-related bleeding. These include appropriate use of alternative dosing and administration strategies (such as fixed dosing and increased infusion speed), adherence to institutional guidelines, and removing significant institutional barriers impacting time to treatment. Clinicians wishing to optimize utilization of 4FPCC for VKA reversal via any of these strategies will hopefully find the results presented here to be of interest.

\section{Authors' Note}

Details of the literature search methodology employed for this review are provided. Publicly available scientific literature was surveyed for this review; therefore, no ethical permissions were required. 


\section{Acknowledgments}

Medical writing support was provided by Lucy Bee, $\mathrm{PhD}$, and Hanna Mourad-Agha, PhD, of Fishawack Communications Ltd, funded by CSL Behring.

\section{Declaration of Conflicting Interests}

The author(s) declared no potential conflicts of interest with respect to the research, authorship, and/or publication of this article.

\section{Funding}

The author(s) disclosed receipt of the following financial support for the research, authorship, and/or publication of this article: DHR has received publication grant funding from CSL Behring. KWC has received research and publication grant funding from CSL Behring. DD has served on an advisory board for CSL Behring.

\section{ORCID iD}

Denise H. Rhoney (D) https://orcid.org/0000-0003-0646-6111

\section{References}

1. Ageno W, Gallus AS, Wittkowsky A, Crowther M, Hylek EM, Palareti G. Oral anticoagulant therapy: antithrombotic therapy and prevention of thrombosis, 9th ed: American College of Chest Physicians Evidence-Based Clinical Practice Guidelines. Chest. 2012;141(2 suppl):e44S-e88S.

2. Quinlan DJ, Eikelboom JW, Weitz JI. Four-factor prothrombin complex concentrate for urgent reversal of vitamin $\mathrm{K}$ antagonists in patients with major bleeding. Circulation. 2013;128(11): 1179-1181.

3. Alarfaj SJ, Jarrell DH, Patanwala AE. Comparison of drug administration logistics between prothrombin complex concentrates and plasma in the emergency department. Am J Emerg Med. 2018; 36(12):2182-2186.

4. Sorensen B, Spahn DR, Innerhofer P, Spannagl M, Rossaint R. Clinical review: prothrombin complex concentrates - evaluation of safety and thrombogenicity. Crit Care. 2011;15(1):201.

5. Keeling D, Baglin T, Tait C, et al. Guidelines on oral anticoagulation with warfarin - fourth edition. Br J Haematol. 2011;154(3): 311-324.

6. Franchini M, Lippi G. Prothrombin complex concentrates: an update. Blood Transfus. 2010;8(3):149-154.

7. Semple JW, Rebetz J, Kapur R. Transfusion-associated circulatory overload and transfusion-related acute lung injury. Blood. 2019;133(17):1840-1853.

8. ASGE Standards of Practice Committee, Acosta RD, Abraham NS, Chandrasekhara V, et al. The management of antithrombotic agents for patients undergoing GI endoscopy. Gastrointest Endosc. 2016;83(1):3-16.

9. Frontera JA, Lewin JJ III, Rabinstein AA, et al. Guideline for reversal of antithrombotics in intracranial hemorrhage: a statement for healthcare professionals from the Neurocritical Care Society and Society of Critical Care Medicine. Neurocrit Care. 2016;24(1):6-46.

10. Holbrook A, Schulman S, Witt DM, et al. Evidence-based management of anticoagulant therapy: antithrombotic therapy and prevention of thrombosis, 9th ed: American College of Chest
Physicians Evidence-Based Clinical Practice Guidelines. Chest. 2012;141(2 suppl):e152S-e184S.

11. Spahn DR, Bouillon B, Cerny V, et al. The European guideline on management of major bleeding and coagulopathy following trauma: fifth edition. Critical Care. 2019;23(1):98.

12. Tomaselli GF, Mahaffey KW, Cuker A, et al. 2017 ACC expert consensus decision pathway on management of bleeding in patients on oral anticoagulants: a report of the American College of Cardiology Task Force on Expert Consensus Decision Pathways. J Am Coll Cardiol. 2017;70(24):3042-3067.

13. Witt DM, Nieuwlaat R, Clark NP, et al. American Society of Hematology 2018 guidelines for management of venous thromboembolism: optimal management of anticoagulation therapy. Blood Adv. 2018;2(22):3257-3291.

14. CSL Behring. KCENTRA ${ }^{\circledR}$ Highlights of Prescribing Information. 2018. https://labeling.cslbehring.com/PI/US/Kcentra/EN/ Kcentra-Prescribing-Information.pdf. Accessed September 9, 2020.

15. Chai-Adisaksopha C, Hillis C, Siegal DM, et al. Prothrombin complex concentrates versus fresh frozen plasma for warfarin reversal. A systematic review and meta-analysis. Thromb Haemost. 2016;116(5):879-890.

16. Chowdary P, Peralta RM. Prothrombin complex concentrates in acquired coagulopathy - friend or foe? Drugs Fut. 2019;44(9): 705 .

17. Goldstein JN, Refaai MA, Milling TJ Jr, et al. Four-factor prothrombin complex concentrate versus plasma for rapid vitamin $\mathrm{K}$ antagonist reversal in patients needing urgent surgical or invasive interventions: a phase 3b, open-label, non-inferiority, randomised trial. Lancet. 2015;385(9982):2077-2087.

18. Hill R, Han TS, Lubomirova I, Math N, Bentley P, Sharma P. Prothrombin complex concentrates are superior to fresh frozen plasma for emergency reversal of vitamin k antagonists: a metaanalysis in 2606 subjects. Drugs. 2019;79(14):1557-1565.

19. Sarode R, Milling TJ Jr, Refaai MA, et al. Efficacy and safety of a 4-factor prothrombin complex concentrate in patients on vitamin $\mathrm{K}$ antagonists presenting with major bleeding: a randomized, plasma-controlled, phase IIIb study. Circulation. 2013;128(11): 1234-1243.

20. Khorsand N, Kooistra HA, van Hest RM, Veeger NJ, Meijer K. A systematic review of prothrombin complex concentrate dosing strategies to reverse vitamin $\mathrm{K}$ antagonist therapy. Thromb Res. 2015;135(1):9-19.

21. Appleby N, Groarke E, Crowley M, et al. Reversal of warfarin anticoagulation using prothrombin complex concentrate at $25 \mathrm{IU}$ $\mathrm{kg}(-1)$ : results of the RAPID study. Transfus Med. 2017;27(1): 66-71.

22. Hall ST, Molina KC. Fixed-dose 4-factor prothrombin complex concentrate: we don't know where we're going if we don't know how to get there. J Thromb Thrombolysis. 2018;46(1):50-57.

23. Klein L, Peters J, Miner J, Gorlin J. Evaluation of fixed dose 4factor prothrombin complex concentrate for emergent warfarin reversal. Am J Emerg Med. 2015;33(9):1213-1218.

24. Astrup G, Sarangarm P, Burnett A. Fixed dose 4-factor prothrombin complex concentrate for the emergent reversal of warfarin: a 
retrospective analysis. $J$ Thromb Thrombolysis. 2018;45(2): 300-305.

25. Varga C, Al-Touri S, Papadoukakis S, Caplan S, Kahn S, Blostein $\mathrm{M}$. The effectiveness and safety of fixed low-dose prothrombin complex concentrates in patients requiring urgent reversal of warfarin. Transfusion. 2013;53(7):1451-1458.

26. Fuh L, Goldstein JN, Hayes BD. Initiation of a fixed-dose fourfactor prothrombin complex concentrate protocol. J Thromb Thrombolysis. 2020;50(1):217-220.

27. Abdoellakhan RA, Miah IP, Khorsand N, Meijer K, Jellema K. Fixed versus variable dosing of prothrombin complex concentrate in vitamin $\mathrm{k}$ antagonist-related intracranial hemorrhage: a retrospective analysis. Neurocrit Care. 2017;26(1):64-69.

28. Khorsand N, Veeger NJ, van Hest RM, Ypma PF, Heidt J, Meijer $\mathrm{K}$. An observational, prospective, two-cohort comparison of a fixed versus variable dosing strategy of prothrombin complex concentrate to counteract vitamin $\mathrm{K}$ antagonists in 240 bleeding emergencies. Haematologica. 2012;97(10):1501-1506.

29. Scott R, Kersten B, Basior J, Nadler M. Evaluation of fixed-dose four-factor prothrombin complex concentrate for emergent warfarin reversal in patients with intracranial hemorrhage. $J$ Emerg Med. 2018;54(6):861-866.

30. Bitonti MT, Rumbarger RL, Absher RK, Curran LM. Prospective evaluation of a fixed-dose 4-factor prothrombin complex concentrate protocol for urgent vitamin $\mathrm{k}$ antagonist reversal. $J$ Emerg Med. 2019;58(2):324-329.

31. Gilbert BW, Morton L, Huffman JB, et al. Modified version of the American College of Cardiology's recommendation for low-dose prothrombin complex concentrate is effective for warfarin reversal. Am J Emerg Med. 2019;38(4):806-809.

32. Butler JJ. Use of low-dose prothrombin complex concentrate before lumbar puncture. Am J Health Syst Pharm. 2015;72(3): 203-205.

33. Zemrak WR, Smith KE, Rolfe SS, et al. Low-dose prothrombin complex concentrate for warfarin-associated intracranial hemorrhage with INR less than 2.0. Neurocrit Care. 2017;27(3): 334-340.

34. Rivosecchi RM, Durkin J, Okonkwo DO, Molyneaux BJ. Safety and efficacy of warfarin reversal with four-factor prothrombin complex concentrate for subtherapeutic INR in intracerebral hemorrhage. Neurocrit Care. 2016;25(3):359-364.

35. Zemrak W, Manuel F, Smith KE, et al. Low-dose compared to manufacturer-recommended dose four-factor prothrombin complex concentrate for acute warfarin reversal. J Thromb Thrombolysis. 2019;47(2):263-271.

36. Wexler AH, Rech MA. Reversal of warfarin-associated lifethreatening bleed with an attenuated dose of 4-factor prothrombin complex concentrate. Ann Pharmacother. 2018;52(2):212-213.

37. Brown CS, Zemrak WR, Dyer KJ, Rolfe S. Low-dose prothrombin complex concentrate in patients with left ventricular assist devices. ASAIO J. 2019;65(2):e21-e24.

38. Gibson G, Owen E, Human T, Wolfe R. Impact of redosing strategies on INR and cerebral bleeding after prothrombin complex concentrate. Crit Care Med. 2016;44(12):290.

39. Malaiyandi DP, Durkin J, Moran C. Safety and efficacy of repeat Kcentra dosing in the neurocritical care population: case series from tertiary care academic center. Neurocritical Care. 2015;23: S1-S289.

40. Yohe AS, Livings SE. Four-factor prothrombin complex concentrate dose response relationship with INR for warfarin reversal. Am J Emerg Med. 2019;37(8):1534-1538.

41. Hirri HM, Green PJ. Audit of warfarin reversal using a new Octaplex reduced dose protocol. Transfus Apher Sci. 2014;51(2): 141-145.

42. Herpers R, van Rossum AP, van Beem RT, et al. INR vs. thrombin generation assays for guiding VKA reversal: a retrospective comparison. Clin Chem Lab Med. 2015;53(8):1227-1236.

43. Miller A, Floroff C, Ingemi A, Tahhan H. Evaluation of an alternate 4-factor prothrombin complex concentrate dosing regimen for warfarin reversal. UNC Eshelman School of Pharmacy Research in Education and Practice Symposium. 2017.

44. Smetana KS, Ziemba R, May CC, Erdman MJ, Van Matre ET, Jones GM. Adjusted versus actual body weight dosing of 4-factor prothrombin complex concentrate in obese patients with warfarinassociated major bleeding. J Thromb Thrombolysis. 2019;47(3): 369-374.

45. Pabinger I, Tiede A, Kalina U, et al. Impact of infusion speed on the safety and effectiveness of prothrombin complex concentrate: a prospective clinical trial of emergency anticoagulation reversal. Ann Hematol. 2010;89(3):309-316.

46. Chester K TM, Barker N. Evaluation of 4-factor prothrombin complex concentrate administered at high infusion rates on patient safety, effcetiveness and turnaround times. Neurocritical Care. 2017;27:S212.

47. Peyko V, Shams D, Urbanski R, Noga J. 4-factor prothrombin complex concentrate administration via intraosseous access for urgent reversal of warfarin. $J$ Emerg Med. 2019;57(1):82-84.

48. Gorlin J, Kinney S, Fung MK, Tinmouth A. Prothrombin complex concentrate for emergent reversal of warfarin: an international survey of hospital protocols. Vox Sang. 2017;112(6):595-597.

49. Desmettre T, Dubart AE, Capellier G, et al. Emergency reversal of anticoagulation: the real use of prothrombin complex concentrates: a prospective multicenter two year French study from 2006 to 2008. Thromb Res. 2012;130(3):e178-183.

50. Duarte RCF, Ferreira CN, Rios DRA, Reis HJD, Carvalho MDG. Thrombin generation assays for global evaluation of the hemostatic system: perspectives and limitations. Rev Bras Hematol Hemoter. 2017;39(3):259-265.

51. Le Roux P, Pollack CV Jr, Milan M, Schaefer A. Race against the clock: overcoming challenges in the management of anticoagulant-associated intracerebral hemorrhage. $J$ Neurosurg. 2014;121(suppl):1-20.

52. Kuramatsu JB, Gerner ST, Schellinger PD, et al. Anticoagulant reversal, blood pressure levels, and anticoagulant resumption in patients with anticoagulation-related intracerebral hemorrhage. JAMA. 2015;313(8):824-836.

53. Tazarourte K, Riou B, Tremey B, Samama CM, Vicaut E, Vigue B. Guideline-concordant administration of prothrombin complex concentrate and vitamin $\mathrm{K}$ is associated with decreased mortality in patients with severe bleeding under vitamin $\mathrm{K}$ antagonist treatment (EPAHK study). Crit Care. 2014;18(2):R81. 
54. Corio JL, Sin JH, Hayes BD, Goldstein JN, Fuh L. Impact of a pharmacist-driven prothrombin complex concentrate protocol on time to administration in patients with warfarin-associated intracranial hemorrhage. West J Emerg Med. 2018;19(5):849-854.

55. Hilbert P, Hofmann GO, Teichmann J, Struck MF, Stuttmann R. The "coagulation box" and a new hemoglobin-driven algorithm for bleeding control in patients with severe multiple traumas. Arch Trauma Res. 2013;2(1):3-10.

56. Parry-Jones A. Cutting delays in reversing anticoagulation after intracerebral haemorrhage: three key changes at a UK comprehensive stroke centre. BMJ Qual Improv Rep. 2015;4(1):u208763. w3521.

57. Stratton M, Grgurich P, Heim K, Mackey S, Burns JD. Feasibility of a collaborative, prospective interdisciplinary review and pharmacy-based dispensing process for prothrombin complex concentrate. Ann Pharmacother. 2018;52(5):454-461.

58. Pavich E, Evans K, Chester K, Lafollette J, Miller S, Johnson S, Vigliotti D. Evaluation of a prothrombin complex concentrate utilization at a large, urban medical center. Critical Care Medicine. 2014;42(12):A1570.

59. Lewis T, Bailey A, Baum R, Schultz A, Thompson S, Justice S. Multicenter retrospective review comparing two different Kcentra dispensing practice. ACCP Annual Meeting 2016. 2016:P102.

60. Jun AH, Chester K, Winkler A, Webb A. Stability of four-factor prothrombin complex concentrate after pneumatic tubing. Crit Care Med. 2016;44(12):298.

61. Masic D, Hidalgo DC, Kuhrau S, Chaney W, Rech MA. Pharmacist presence decreases time to prothrombin complex concentrate in emergency department patients with lifethreatening bleeding and urgent procedures. J Emerg Med. 2019;57(5):620-628.

62. Vitt JR, Do LV, Shah NH, Fong G, Nguyen NY, Kim AS. Beforeafter study of an electronic order set for reversal of vitamin $\mathrm{k}$ antagonist-associated intracerebral hemorrhage. Neurohospitalist. 2018;8(1):18-23.

63. Vines C, Tesseneer SJ, Cox RD, Darsey DA, Carbrey K, Puskarich MA. Air ambulance delivery and administration of four-factor prothrombin complex concentrate is feasible and decreases time to anticoagulation reversal. Acad Emerg Med. 2018;25(1):33-40.

64. Gomes JA, Ahrens CL, Hussain MS, Winners S, Rasmussen PA, Uchino K. Prehospital reversal of warfarin-related coagulopathy in intracerebral hemorrhage in a mobile stroke treatment unit. Stroke. 2015;46(5):e118-120.

65. Robertson LC, McKinlay JA, Munro PT, Hearns S. Use of prothrombin complex concentrates: 4-year experience of a national aeromedical retrieval service servicing remote and rural areas. Emerg Med J. 2014;31(2):109-114.

66. Faine BA, Amendola J, Homan J, Ahmed A, Mohr N. Factors associated with availability of anticoagulation reversal agents in rural and community emergency departments. Am J Health Syst Pharm. 2018;75(2):72-77.

67. Lendrum RA, Kotze JP, Lockey DJ, Weaver AE. Case studies in prehospital care from London HEMS: pre-hospital administration of prothrombin complex concentrate to the head-injured patient. Emerg Med J. 2013;30(3):247-248.

68. Zepeski A, Rewitzer S, Leira EC, Harland K, Faine BA. Interhospital transfer delays anticoagulation reversal in warfarinassociated intracranial hemorrhage. J Stroke Cerebrovasc Dis. 2018;27(11):3345-3349.

69. Unold D, Tormey CA. Clinical applications of 4-factor prothrombin complex concentrate: a practical pathologist's perspective. Arch Pathol Lab Med. 2015;139(12):1568-1575.

70. Bernardi E, Imberti D, Ferrari A. Management strategies for vitamin $\mathrm{k}$ antagonists reversal in patients with major bleeding: a survey of Italian emergency departments. Clin Appl Thromb Hemost. 2017;23(1):40-44.

71. Bordeleau S, Poitras J, Marceau D, Breton C, Beaupre P, Archambault PM. Use of prothrombin complex concentrate in warfarin anticoagulation reversal in the emergency department: a quality improvement study of administration delays. BMC Health Serv Res. 2015;15:106.

72. Hanger C, Geddes J, Wilkinson T, et al. Improving the use and timeliness of anticoagulation reversal for warfarin related intracranial haemorrhage. N Z Med J. 2016;129(1445):35-49.

73. Wanek MR, Hodges K, Persaud RA, et al. Prothrombin complex concentrates for warfarin reversal before heart transplantation. Ann Thorac Surg. 2019;107(5):1409-1415.

74. Khorsand N, Giepmans L, Meijer K, van Hest RM, Veeger NJ. A low fixed dose of prothrombin complex concentrate is cost effective in emergency reversal of vitamin $\mathrm{K}$ antagonists. Haematologica. 2013;98(6):e65-67.

75. Schwebach AA, Waybright RA, Johnson TJ. Fixed-dose fourfactor prothrombin complex concentrate for vitamin $\mathrm{k}$ antagonist reversal: does one dose fit all? Pharmacotherapy. 2019;39(5): 599-608.

76. Abdoellakhan RA, Khorsand N, Van Hest RM, et al. Randomised controlled trial protocol to evaluate a fixed dose prothrombin complex concentrate against the variable dose in vitamin $\mathrm{K}$ antagonist related bleeding (PROPER3). BMJ Open. 2018;8(3): e020764. 